\title{
Cost-Utility Analysis of Dapagliflozin as an Add-on to Standard of Care for Patients with Chronic Kidney Disease in Thailand
}

\author{
Kriengsak Vareesangthip · Chaicharn Deerochanawong • \\ Dittaya Thongsuk $\cdot$ Nuch Pojchaijongdee $\cdot$ Unchalee Permsuwan
}

Received: November 24, 2021 / Accepted: December 22, 2021 / Published online: January 17, 2022

(c) The Author(s) 2022

\begin{abstract}
Introduction: Chronic kidney disease (CKD) creates a significant economic burden on patients and society. The DAPA-CKD trial reports the benefit of dapagliflozin in CKD patients; however, its cost-effectiveness is unknown in Thailand. This study evaluated the cost-utility of dapagliflozin in addition to standard of care (SoC) compared with SoC alone in CKD patients.

Methods: A Markov model was employed to estimate lifetime costs, life-years, and qualityadjusted life-year (QALY), with the modeled
\end{abstract}

\section{K. Vareesangthip}

Renal Division, Department of Medicine, Faculty of Medicine Siriraj Hospital, Mahidol University,

Bangkok 10700, Thailand

C. Deerochanawong

Rajavithi Hospital, College of Medicine, Rangsit University, Ministry of Public Health, Bangkok 10400, Thailand

\section{Thongsuk}

Market Access and Government Affairs,

AstraZeneca, Bangkok, Thailand

N. Pojchaijongdee

Medical Affairs, AstraZeneca, Bangkok, Thailand

U. Permsuwan $(\bowtie)$

Department of Pharmaceutical Care, Faculty of

Pharmacy, Chiang Mai University, Chiang Mai

50200, Thailand

e-mail: unchalee.permsuwan@gmail.com population aligned to the baseline characteristics of a DAPA-CKD trial, from a societal perspective. Effectiveness inputs were obtained from the DAPA-CKD trial. Costs and most utility data were gathered from published studies conducted in Thailand. Costs and benefits were discounted at 3\% per annum. A series of sensitivity analyses were performed.

Results: Over a lifetime horizon, add-on dapagliflozin was estimated to increase life-years by 0.34 and QALY by 0.30 in comparison with SoC alone (7.13 vs. 6.78 years, 5.10 vs. 4.80 QALYs). Total cost was lower under dapagliflozin treatment than SoC treatment $(648,413$ THB vs. 689,284 THB or $20,947.64$ USD vs. $22,268.01$ USD). Cost saving occurred as a result of the lower costs of dialysis and KT. The findings were robust to the changes of inputs.

Conclusions: On the basis of the DAPA-CKD trial, the add-on dapagliflozin results in cost saving compared favorably with SoC alone in Thailand. The benefit of dapagliflozin in delayed CKD progression is that it reduces the requirement for dialysis and $\mathrm{KT}$, which can offset the costs of dapagliflozin and early CKD treatment.

Keywords: Chronic kidney disease; Cost-utility analysis; Cost-effectiveness analysis; Dapagliflozin; SGLT-2 inhibitor 


\section{Key Summary Points}

Clinical evidence from RCT confirms the benefit of dapagliflozin in CKD patients. Cost is a major concern, especially for middle- or low-income countries.

The findings of the cost-utility study reveals that the benefit accrued from addon dapagliflozin can offset its acquisition cost.

SGLT-2 inhibitor should be considered as the treatment for T2DM with CKD patients as the guideline recommendation. The data also support SGLT-2 inhibitor as a potential option for CKD patients without T2DM.

\section{INTRODUCTION}

Chronic kidney disease (CKD) was ranked 27th in the cause of death in 1990, and rose to 18th in 2010 in the 2010 Global Burden of Disease study [1]. It is one of the most significant global burdens on public health worldwide, with a prevalence of $8-16 \%$, and was found to be associated with increased morbidity and mortality [2]. As the kidney function progressively declines, patients transition to renal replacement therapy (RRT). In 2010, the estimated number of patients on dialysis was more than 2 million and was predicted to double by 2030 [3]. The mean annual total health cost also increases as the CKD progresses, with the highest cost for patients with CKD stage 5 with renal replacement therapy [4].

The efficacy of dapagliflozin as a treatment for CKD when used in addition to standard of care (SoC) was assessed in the Dapagliflozin and Prevention of Adverse Outcomes in Chronic Kidney Disease (DAPA-CKD) clinical trial (NCT03036150) [5]. The findings of the study showed that dapagliflozin was associated with a significant reduction in the incidence of the primary composite endpoint of a sustained $\geq 50 \%$ decline in the estimated glomerular filtration rate (eGFR), end stage renal disease (ESRD), cardiovascular death, and renal death (hazard ratio 0.61, 95\% confidence interval 0.51-0.72) in comparison with SoC.

In addition to clinical evidence, economic evaluation, such as cost-utility analysis, has been used as a tool in many countries, including Thailand, to generate useful financial evidence to allocate limited healthcare resources more efficiently by comparing the costs and outcomes of various treatment strategies. Hence, this study aimed to assess the cost-utility of adding dapagliflozin to SoC compared with SoC alone for the treatment of CKD over a lifetime horizon from a societal perspective.

\section{METHODS}

\section{Cohort Population}

The cohort population reflects the participants included in the DAPA-CKD clinical trial. [5] In brief, the eligible patients were adults with or without type 2 diabetes who had an eGFR of 25-75 mL per min per $1.73 \mathrm{~m}^{2}$ of body surface area, and a urinary albumin-to-creatinine ratio of $200-5000 \mathrm{mg} / \mathrm{g}$. The starting age in this study was 60 years.

\section{Compliance with Ethics Guidelines}

This study was based on previously conducted studies and did not include any new studies with human participants or animals performed by any of the authors, and was not registered with any clinical trial database.

\section{Intervention and Comparator}

The intervention in this study was dapagliflozin (10 mg once daily) as an add-on to the current background therapy or SoC. The SoC was to maintain patients with a stable optimized dose of either an angiotensin-converting enzyme inhibitor (ACEI) or an angiotensin receptor blocker (ARB) Patients in the dapagliflozin group received an optimized dose of either ACEI or ARB similar to those in the SoC group. 


\section{Model Structure}

A lifetime Markov state transition model with a 1-month cycle was employed based on the clinical evidence from the DAPA-CKD clinical trial [5]. The model health states were defined by CKD state from stage 1 to stage 5 and ESRD which was stratified into dialysis and transplant on the basis of progression events observed in the DAPA-CKD clinical trial (Fig. 1). The cohort population was distributed across all available CKD stages at baseline, consistent with the baseline population characteristic of the DAPACKD clinical trial, which were $11 \%, 31 \%, 44 \%$, and $14 \%$ in CKD stage 2 , stage $3 a$, stage $3 b$, and stage 4 , respectively [5]. There were no patients in CKD stage 1 , stage 5 , or dialysis at the beginning. Patients would get worse and move to a more advanced CKD stage or regress and move back to a lower CKD stage depending on

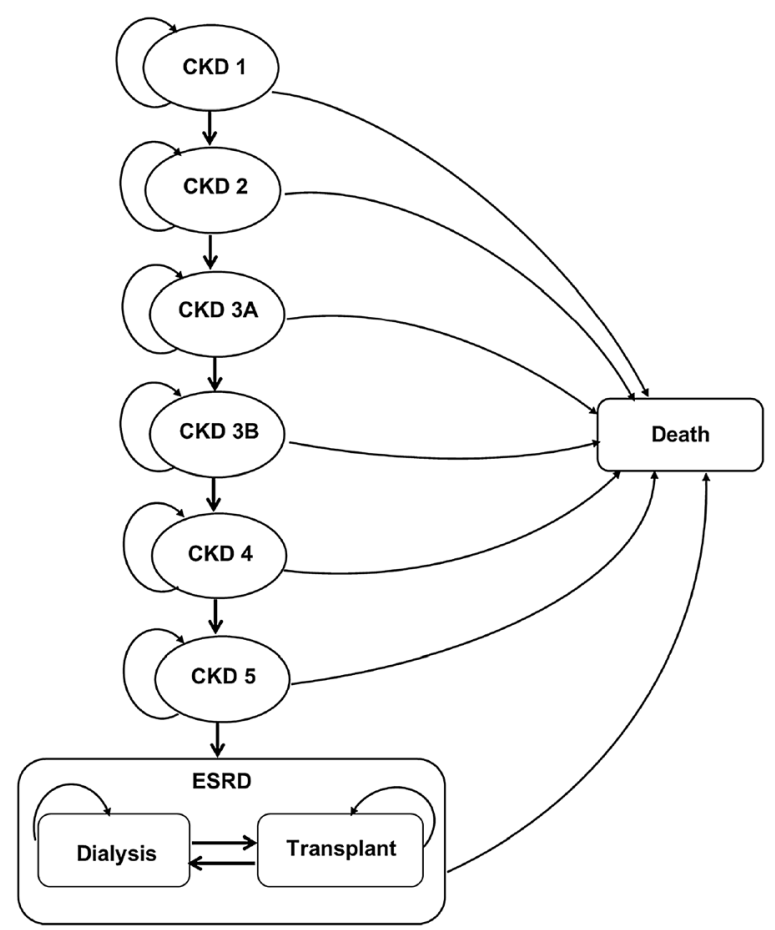

Fig. 1 Markov model of patients with chronic kidney disease. CKD 1 chronic kidney disease stage 1, CKD 2 chronic kidney disease stage 2, CKD $3 a$ chronic kidney disease stage $3 \mathrm{a}, C K D 3 b$ chronic kidney disease stage $3 \mathrm{~b}$, CKD 4 chronic kidney disease stage 4, CKD 5 chronic kidney disease stage 5, ESRD end stage renal disease the transitional probabilities. When patients moved to the ESRD stage with RRT, they were not able to move back to the pre-RRT stage. All patients eventually would enter the absorbing health state, which is the death state.

\section{Input Parameters}

\section{Mortality}

On the basis of the findings from the DAPACKD clinical trial [5], the overall death rate of patients in the dapagliflozin group and the SoC group was 2.2 and 3.1 events per 100 patientyear, respectively. These rates were converted to risk using the formula $p=1-\exp (-r t)$, where $p$ is probability, $r$ is the rate, and $t$ is the duration. The 1-month mortality risk was 0.002 and 0.003 for dapagliflozin and SoC treatment, respectively.

In addition, we applied the age-specific mortality rate (ASMR) of the Thai population into the model. The Thai ASMR was adjusted for the increased excess risk of death from the CKD status. The adjusted hazard ratio (HR) for death from any cause in patients with CKD aged $\geq 65$ years was $4.51 \quad[95 \%$ confidence interval (CI) 3.95-5.15] [6]. To estimate the mortality rate of patients with CKD in Thailand, the ASMR of the Thai population, excluding renal diseases, with data from the Ministry of Public Health [7], was multiplied by this HR. Next, we converted the rate to 1 -month risk.

\section{Health State Transition for Chronic Kidney Disease}

The transitional probabilities between CKD stages were derived from the DAPA-CKD clinical trial [5]. Transitions were split into months $0-4$ and month 4 onwards to capture the change in trend observed in mean eGFR in the DAPA-CKD clinical trial [5]. This is as a result of the association of dapagliflozin with an initial decrease in eGFR, following by a nominal increase over the first 4 months of the trial. After a 4-month period, the trends for both treatment groups were approximated by straight lines. Therefore, independent transitional probabilities were derived based on the first 4 months of the DAPA-CKD clinical trial to account for the 


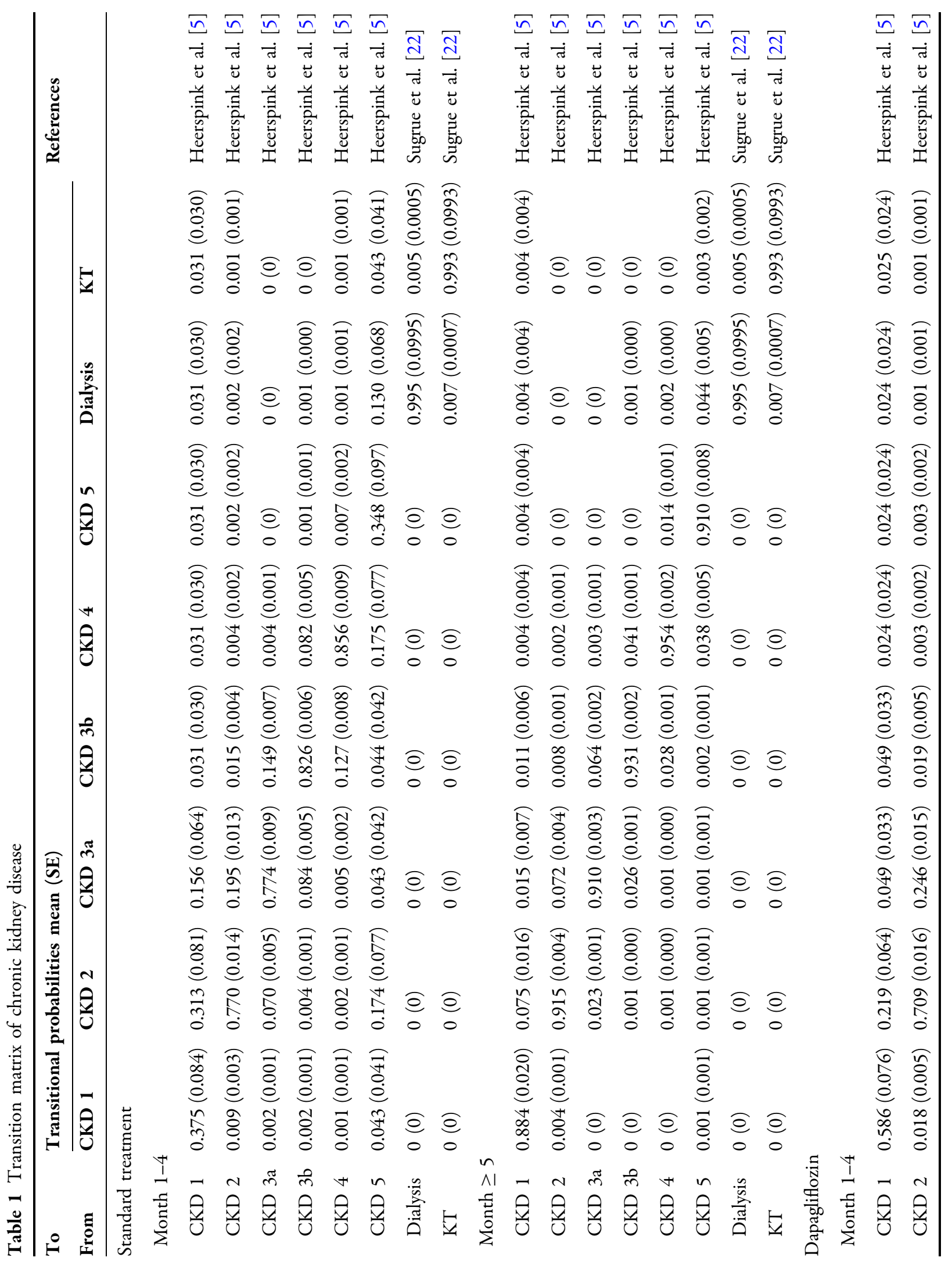




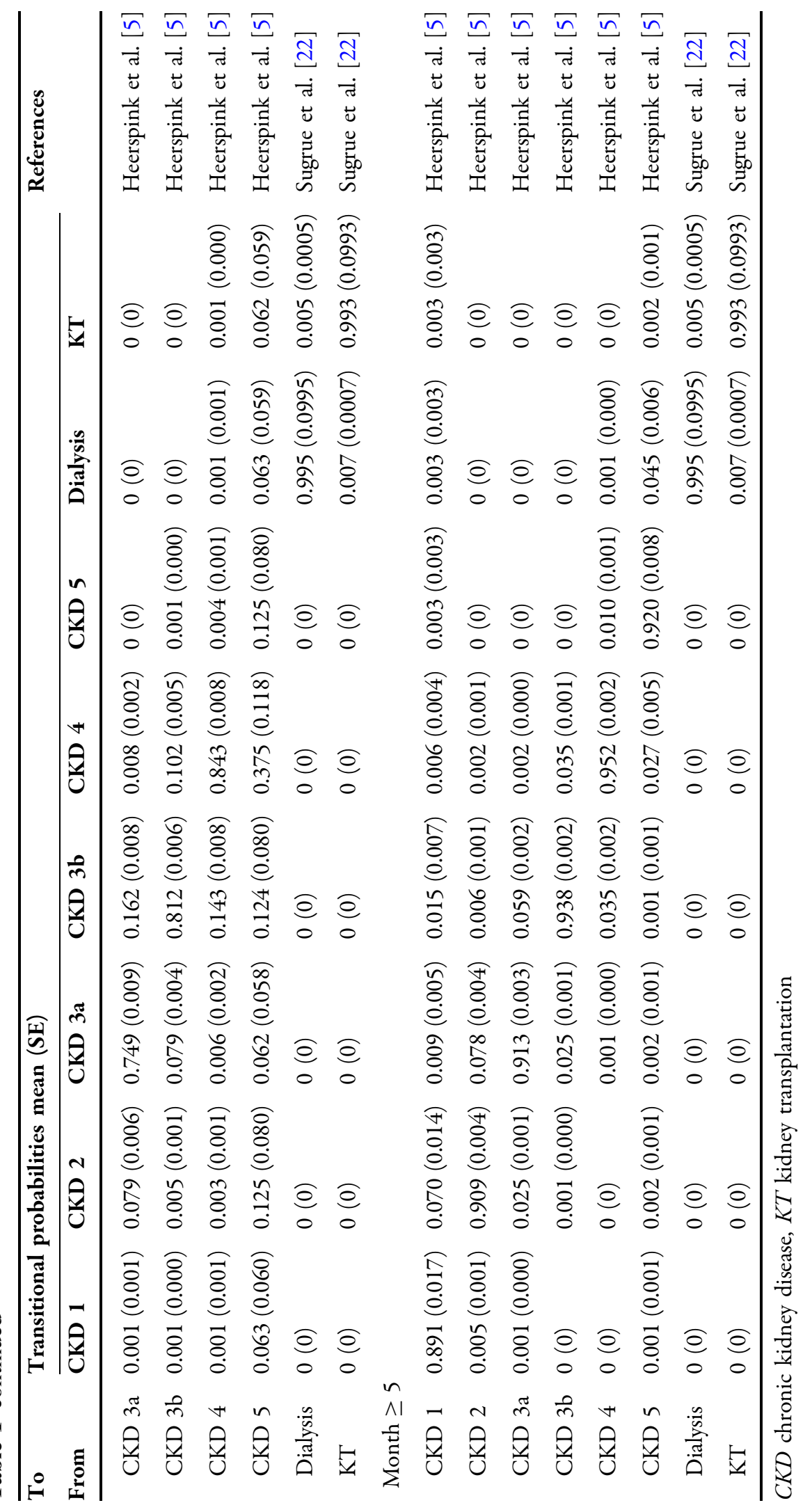


Table 2 Cost and utility data

\begin{tabular}{|c|c|c|c|c|}
\hline Parameter & Value & Range & Distribution & References \\
\hline \multicolumn{5}{|c|}{ Costs of CKD treatment [THB (USD) per month] } \\
\hline CKD stage 1 & $675(21.79)$ & $540-810(17.44-26.15)$ & Gamma & Cha'on et al. [12] \\
\hline CKD stage 2 & $749(24.19)$ & $599-898(19.35-29.02)$ & Gamma & Cha'on et al. [12] \\
\hline CKD stage 3 & $\begin{array}{l}2488 \\
\quad(80.37)\end{array}$ & $\begin{array}{l}1990-2985 \\
\quad(64.30-96.45)\end{array}$ & Gamma & Cha'on et al. [12] \\
\hline CKD stage 4 & $\begin{array}{l}2733 \\
\quad(88.29)\end{array}$ & $\begin{array}{l}2186-3279 \\
\quad(70.63-105.94)\end{array}$ & Gamma & Cha'on et al. [12] \\
\hline CKD stage 5 & $\begin{array}{l}3535 \\
\quad(114.19)\end{array}$ & $\begin{array}{l}2828-4242 \\
\quad(91.35-137.03)\end{array}$ & Gamma & Cha'on et al. [12] \\
\hline Dialysis, first year & $\begin{array}{l}38,991 \\
\quad(1259.65)\end{array}$ & $\begin{array}{l}31,193-46,790 \\
\quad(1007.72-1511.58)\end{array}$ & Gamma & $\begin{array}{l}\text { Permsuwan et al. [13], } \\
\text { Nephrology Society [14] }\end{array}$ \\
\hline Dialysis, second year onward & $\begin{array}{l}36,425 \\
\quad(1176.73)\end{array}$ & $\begin{array}{l}29,140-43,709 \\
\quad(941.38-1412.08)\end{array}$ & Gamma & $\begin{array}{l}\text { Permsuwan et al. [13], } \\
\text { Nephrology Society [14] }\end{array}$ \\
\hline $\begin{array}{l}\text { Kidney transplantation, first } \\
\text { year }\end{array}$ & $\begin{array}{l}79,735 \\
\quad(2575.92)\end{array}$ & $\begin{array}{l}63,788-95,682 \\
\quad(2060.74-3091.11)\end{array}$ & Gamma & Permsuwan et al. [13] \\
\hline $\begin{array}{l}\text { Kidney transplantation, } \\
\text { second year onward }\end{array}$ & $\begin{array}{l}36,881 \\
\quad(1191.47)\end{array}$ & $\begin{array}{l}29,505-44,257 \\
\quad(953.18-1429.77)\end{array}$ & Gamma & Permsuwan et al. [13] \\
\hline \multicolumn{5}{|c|}{ Cost of adverse event treatment (THB (USD) per event) } \\
\hline Major hypoglycemia & $\begin{array}{l}51,298 \\
\quad(1657.23)\end{array}$ & $\begin{array}{l}2331-100,264 \\
\quad(75.32-3239.13)\end{array}$ & Gamma & Srinonprasert et al. [11] \\
\hline Volume depletion & $\begin{array}{l}22,960 \\
(741.74)\end{array}$ & $\begin{array}{l}20,858-25,062 \\
\quad(673.83-809.66)\end{array}$ & Gamma & Krittayaphong et al. $[10]$ \\
\hline \multicolumn{5}{|c|}{ Cost of drug (THB (USD) per month) } \\
\hline Dapagliflozin & $\begin{array}{l}1220 \\
(39.41)\end{array}$ & $\begin{array}{l}976-1464 \\
\quad(31.53-47.29)\end{array}$ & Gamma & DMSIC [9] \\
\hline \multicolumn{5}{|c|}{ Direct non-medical cost (THB (USD) per month) } \\
\hline $\begin{array}{l}\text { Food and travel costs for non- } \\
\text { dialysis stage }\end{array}$ & 88 & $75-101$ & Gamma & Srisubat et al. [15] \\
\hline $\begin{array}{l}\text { Food and travel costs for } \\
\text { dialysis stage }\end{array}$ & 708 & $536-880$ & Gamma & Srisubat et al. [16] \\
\hline \multicolumn{5}{|l|}{ Utility } \\
\hline CKD stage 1 & 0.85 & $0.76-0.94$ & Beta & Jesky et al. [17] \\
\hline CKD stage 2 & 0.85 & $0.76-0.94$ & Beta & Jesky et al. [17] \\
\hline CKD stage $3 a$ & 0.72 & $0.57-0.87$ & Beta & Srisubat et al. [15] \\
\hline CKD stage $3 b$ & 0.72 & $0.57-0.87$ & Beta & Srisubat et al. [15] \\
\hline
\end{tabular}


Table 2 continued

\begin{tabular}{lllll}
\hline Parameter & Value & Range & Distribution & References \\
\hline CKD stage 4 & 0.72 & $0.57-0.87$ & Beta & Srisubat et al. [15] \\
CKD stage 5 & 0.70 & $0.63-0.77$ & Beta & Srisubat et al. [15] \\
Dialysis & 0.55 & $0.50-0.60$ & Beta & Srisubat et al. [16] \\
Kidney transplantation & 0.83 & $0.75-0.91$ & Beta & Li et al. [18] \\
\hline
\end{tabular}

CKD chronic kidney disease, DMSIC Drug and Medical Supply Information Center

Table 3 Results from base case analysis

\begin{tabular}{llll}
\hline Outcome & Dapagliflozin & Standard care & Incremental \\
\hline Lifetime cost THB (USD) & $648,413(20,947.64)$ & $689,284(22,268.01)$ & $-40,871(-1320.37)$ \\
CKD stage 1 & $834(26.94)$ & $170(5.50)$ & $664(21.44)$ \\
CKD stage 2 & $11,201(361.86)$ & $4,354(140.65)$ & $6,847(221.21)$ \\
CKD stage 3a & $57,982(1873.18)$ & $33,879(1094.50)$ & $24,103(778.68)$ \\
CKD stage 3b & $115,300(3724.88)$ & $63,168(2040.72)$ & $52,132(1684.16)$ \\
CKD stage 4 & $94,828(3063.52)$ & $65,187(2105.94)$ & $29,641(957.57)$ \\
CKD stage 5 & $12,217(394.67)$ & $11,305(365.21)$ & $912(29.45)$ \\
Dialysis & $284,151(9179.80)$ & $409,638(13,233.76)$ & $-125,486(-4,053.96)$ \\
Kidney transplantation & $71,900(2322.79)$ & $101,583(3281.73)$ & $-29,683(-958.93)$ \\
Life-years & 7.13 & 6.78 & 0.34 \\
Quality-adjusted life-years & 5.10 & 4.80 & 0.30 \\
Incremental cost-effectiveness ratio $(\mathrm{THB} /$ life-year) & & Cost-saving \\
Incremental cost-effectiveness ratio (THB/QALY) & & Cost-saving \\
\hline
\end{tabular}

$C K D$ chronic kidney disease, $Q A L Y$ quality-adjusted life-year, $T H B$ Thai Baht

initial decline in eGFR observed in patients treated with dapagliflozin. A second set of transitional probabilities was applied from month 5 onwards to capture the long-term trend. All health state transitions are shown in Table 1.

\section{Adverse Events}

The most significant adverse events reported in the DAPA-CKD clinical trial were major hypoglycemia and volume depletion. Both adverse events were included for data analysis. Patients with dapagliflozin treatment had higher events of volume depletion but lower major hypoglycemia than those with SoC treatment $(5.9 \%$ vs. $4.2 \%, 0.7 \%$ vs. $1.3 \%$, respectively) [5]. 


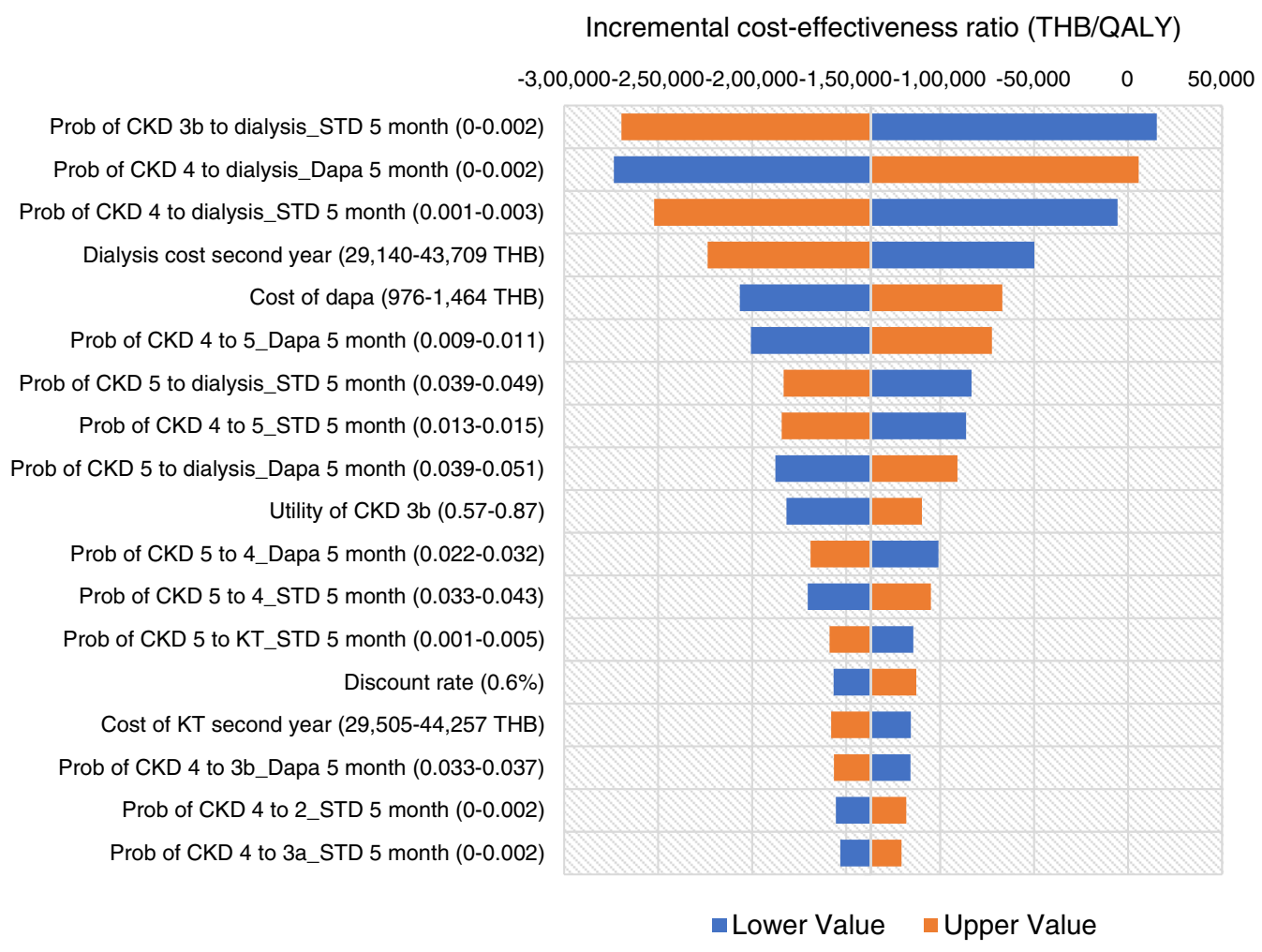

Fig. 2 Tornado diagram for the cost-utility analysis of an add-on dapagliflozin and standard of care for patients with CKD from a societal perspective over a lifetime horizon. CKD $3 a$ chronic kidney disease stage $3 a, C K D 3 b$ chronic kidney disease stage $3 \mathrm{~b}, C K D 4$ chronic kidney disease

stage 4, CKD 5 chronic kidney disease stage 5, Dapa dapagliflozin, ICER incremental cost-effectiveness ratio, KT kidney transplantation, Prob probability, $Q A L Y$ quality-adjusted life-year, $T H B$ Thai Baht

\section{Costs}

The Thai Health Technology Assessment (HTA) guideline recommends the use of a societal perspective for economic evaluation [8]. Direct medical costs and direct non-medical costs were included for analysis; however, indirect costs were excluded for the cost-utility analysis to avoid double counting of both the cost and the effect of the interventions. Those included the acquisition cost of dapagliflozin, the cost of CKD treatment, and the cost of adverse event treatment and direct non-medical costs, such as food and travel. In addition, we assumed that other direct costs related to outpatient visits, such as laboratories, monitoring, the cost of standard treatment, and the like, were not dependent on whether CKD patients received SoC treatment or add-on dapagliflozin treatment. As a result, those direct costs were

cancelled out when the incremental cost was calculated.

The cost of dapagliflozin was obtained from the Drug and Medical Supply Information Center (DMSIC), Ministry of Public Health [9]. The HTA guideline recommends using the median drug price. The total cost per month for dapagliflozin was calculated based on daily dose and unit cost. The daily dose used in this study was similar to the dose used in the DAPA-CKD clinical trial [5]. The median price of dapagliflozin was 1219.80 THB (39.41 USD) per month.

In this study, only significant adverse events reported in the DAPA-CKD clinical trial were included for data analyses, which were major hypoglycemia and volume depletion. The costs of adverse event treatment were obtained from published Thai studies $[10,11]$, which obtained the costs from the Siriraj hospital database. The 


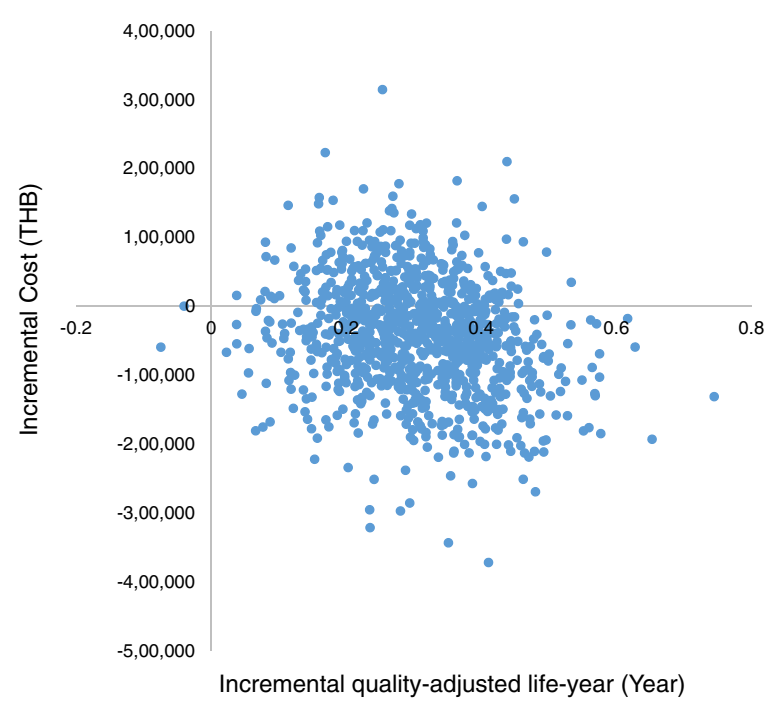

Fig. 3 Scatter plot of 1000 iterations of incremental cost and incremental QALY between an add-on dapagliflozin treatment compared with standard treatment for patients with chronic kidney disease on the cost-effectiveness plane

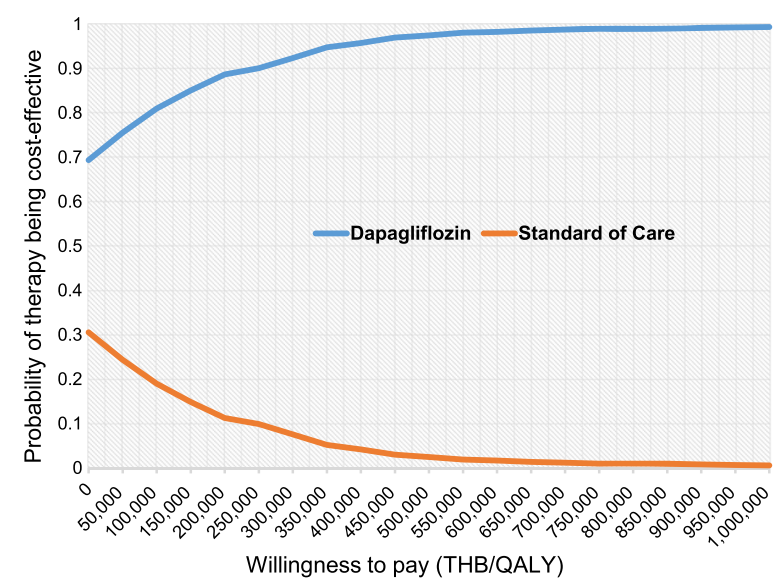

Fig. 4 Cost-effectiveness acceptability curve of an add-on dapagliflozin treatment compared with standard of care treatment

cost of major hypoglycemia treatment was 51,298 THB per event (1657.23 USD) and the cost of volume depletion treatment was 22,960 THB per event (741.74 USD).

The costs of CKD treatment were obtained from the Chronic Kidney Disease Prevention in the Northeast Thailand (CKDNET) study [12]. In brief, the CKDNET has been conducted at the Faculty of Medicine, Khon Kaen University, in collaboration with the public health sector since 2016. It has been established for activities such as screening, surveillance, diagnosis, treatment, awareness, and the management of kidney disease. The monthly costs of CKD stage 1 , CKD stage 2 , CKD stage 3 , CKD stage 4 , and CKD stage 5 were 675 THB (21.79 USD), 749 THB (24.19 USD), 2488 THB (80.37 USD), 2733 THB (88.29 USD), and 3535 THB (114.19 USD), respectively.

The dialysis cost was the sum of the hemodialysis (HD) cost and HD usage and the peritoneal dialysis (PD) cost and PD usage. The costs of $\mathrm{HD}, \mathrm{PD}$, and kidney transplantation were obtained from the historical cost-effectiveness of dipeptidyl peptidase- 4 inhibitor in patients with type 2 diabetes and CKD in Thailand [13]. The proportion of HD or PD usage was derived from the Annual Report Thailand Renal Replacement Therapy [14]. The prevalence of HD and PD from 2017 to 2019 was about $79 \%$ and $21 \%$, respectively. The yearly costs of HD and PD were 466,162 THB $(15,059.82$ USD) and 474,419 THB $(15,326.59$ USD) in the first year and 441,438 THB $(14,261.09$ USD) and 420,754 THB (13,592.87 USD) in the second year. The yearly cost of dialysis was 467,896 THB $(466,162 \times 79 \%+474,419 \times 21 \%)$ in the first year and 437,094 THB $(441,438 \times 79 \%+420,754 \times 21 \%)$ in the second year. Therefore, the cost of dialysis was 38,991 THB per month (1259.65 USD) for 12 months, followed by 36,425 THB per month (1176.73 USD) in month 13 and onward.

The direct non-medical costs were obtained from the studies conducted on Thai patients with CKD disease $[15,16]$. All cost data are shown in Table 2.

All costs were adjusted for inflation based on the medical care section of Thailand's consumer price index and presented in 2020. The costs were converted into USD at a rate of $30.95 \mathrm{THB}$ per USD, as of 1 July 2020.

\section{Utility}

The utility data of Thai CKD patients would be the priority for data selection. The study conducted by Srisubat et al. reported the utilities of Thai patients with CKD stages 3-5 and dialysis measured by EuroQoL, which were 0.72, 0.72, 
0.70 , and 0.55 , respectively $[15,16]$. Due to a paucity of utility data for patients with CKD stages 1 and 2, and kidney transplantation, we obtained the utility data from published studies $[17,18]$. All utility data are shown in Table 2.

\section{Analyses and Outcomes}

The Markov model was built into Microsoft Excel 2010 (Microsoft, Redmond, WA, USA). The predicted long-term outcomes and costs were estimated and discounted at $3 \%$, which is recommended by the HTA guideline [19]. The incremental cost-effectiveness ratio (ICER) was calculated using the formula ICER $=($ total cost of dapagliflozin group - total cost of SoC group)/(effect of dapagliflozin group - effect of SoC group), where effect is either life-year or quality-adjusted life-year (QALY). When the estimated ICER is below the acceptable local willingness-to-pay (WTP) threshold of 160,000 THB/QALY (5168.96 USD/QALY) [20], the new intervention is justified in terms of costeffectiveness.

\section{Sensitivity Analyses}

To assess the robustness of the base-case results to changes in the input parameters, one-way sensitivity analysis, scenario analysis, and probabilistic sensitivity analysis (PSA) were conducted. For one-way sensitivity analysis, all probabilities, costs, and utilities (169 parameters) were varied within a range of standard error (SE). When SE was not available, probability and utility were varied by $\pm 10 \%$ and the cost was varied by $\pm 20 \%$. The results are displayed as a tornado diagram.

The scenario analysis was performed to present the results from adding treatment costs of minor events, such as urinary tract infection (UTI) and genital infection. The findings from the DAPA-CKD clinical trial [5] reported 20 versus 10 UTI events and 1 versus o genital event in the dapagliflozin group compared with the SoC group, respectively. Treatment costs of UTI and genital infection were 53,939 THB (1742.56 USD) and 11 THB (0.36 USD), respectively.
The PSA employed the recommended distributions by Briggs et al. [21]. Beta distribution is appropriate for transitional probability and utility due to the range of $0-4$. Gamma distribution is appropriate for cost data owing to the positive value. The model parameters were randomly sampled (1000 samples) based on their distribution. The results are presented as a scatter plot on the cost-effectiveness plane. In addition, the probabilities of the new intervention being cost-effective for different WTP thresholds are presented in a cost-effectiveness acceptability curve.

\section{Study Process}

A literature search for the CKD model was conducted. The core model structure of the CKD model based on the systematic review of economic modeling of CKD [22] was adopted. We established two consultation panels. The first panel aimed to finalize the scope of the study, check the input parameters, and crosscheck the face validity of the adopted model to ensure that the model was consistent with the history of the disease and practical in a local context. The second panel aimed to assess the preliminary results of the study. All suggestions were taken into consideration.

\section{RESULTS}

\section{Base Case Result}

It was found that the lifetime total cost of the add-on dapagliflozin group was lower than that of the SoC group $(648,413$ THB vs. 689,284 THB or 20,947.64 USD vs. 22,268.01 USD) as shown in Table 3. When total costs were disaggregated, much lower cost was incurred for dialysis and kidney transplantation for the add-on dapagliflozin group than the SoC group. Among the pre-dialysis stages, patients with CKD stage $3 \mathrm{~b}$ in the add-on dapagliflozin group had the greatest difference in total cost compared with the SoC group. In addition, CKD patients with dapagliflozin treatment gained slightly more life-years and QALYs than those with SoC 
treatment (7.13 vs. 6.78 and 5.10 vs. 4.80 , respectively). Therefore, the ICER would become negative with less cost and better outcomes for dapagliflozin compared with SoC treatment (Table 3).

\section{Sensitivity Analyses}

When 169 individual parameters were varied within a specified range, all estimated ICERs were negative, except for the lower value of probability of CKD stage $3 \mathrm{~b}$ to dialysis of SoC at month 5 (ICER $=15,272$ THB/QALY or 493.39 USD/QALY), and the upper value of probability of CKD stage 4 to dialysis of dapagliflozin at month 5 (ICER $=5568$ THB/QALY or 179.89 USD/QALY). The negative ICER represented a cost-saving of add-on dapagliflozin resulting from lower cost with higher QALY compared with the standard treatment (Fig. 2).

Adding the treatment costs of minor adverse event slightly increased the lifetime total cost of the dapagliflozin group and the SoC group $(649,910$ THB vs. 690,351 THB or $20,996.01$ USD vs. $22,302.50$ USD). The lifetime total cost of the add-on dapagliflozin group was still lower than that of the SoC group. However, the magnitude of total cost saved was slightly less than the base case analysis $(40,441$ THB vs. 40,871 THB or 1306.49 USD vs. 1320.37 USD).

Of all 1000 iterations, about 70\% fell in the lower right quadrant, indicating that an add-on dapagliflozin incurred less lifetime total cost and gained more QALYs (Fig. 3). Figure 4 shows that add-on dapagliflozin treatment was a costeffective strategy at all levels of WTP.

\section{DISCUSSION}

ACEIs and ARBs are currently the SoC for delaying CKD progression, but CKD patients still face a substantial residual risk of progression towards ESRD and mortality [23, 24]. Consequently, there remains a need for treatment which can preserve kidney function, slow disease progression, and reduce mortality in patients with CKD.

This is the first cost-utility study that has investigated the value-for-money of dapagliflozin, a sodium-glucose co-transporter2 (SGLT2) inhibitor in combination with SoC, when compared with SoC alone in CKD patients under a societal perspective in Thailand. The findings of the study show that CKD patients treated with dapagliflozin would have less lifetime total cost and slightly gain lifeyears and QALYs compared with SoC alone $(648,416$ THB vs. 689,284 THB or $20,947.64$ USD vs. $22,268.01$ USD; 7.13 vs. 6.78 years; 5.10 vs. 4.80 QALYs, respectively). The cost-saving benefit of adding dapagliflozin results from lower dialysis and kidney transplantation costs compared with SoC alone $(-125,486$ THB or -4053.96 USD; $-29,683$ THB or -958.93 USD, respectively). The monthly cost of dialysis and kidney transplantation was much higher than that of pre-RRT. The benefits associated with delayed CKD progression to dialysis from dapagliflozin are a potential reduction in the economic burden of CKD treatment.

The results from varying individual parameters from 169 parameters when conducting oneway sensitivity analysis also confirmed the costsavings of add-on dapagliflozin. Only two parameters, the probability of CKD stage $3 b$ to dialysis in the SoC group at month 5 and the probability of CKD stage 4 to dialysis in the dapagliflozin group at month 5 , had positive ICER. However, the positive ICER fell below the acceptable local threshold of $160,000 \mathrm{THB} /$ QALY (5168.96 USD/QALY) [20]. In addition, add-on dapagliflozin was a cost-effective alternative at all thresholds of the WTP based on the findings from the PSA.

According to the subgroup analysis of the DAPA-CKD clinical trial [25], the event rate of first hospitalization for heart failure (HHF) and cardiovascular death was lower in the dapagliflozin group than in the SoC group (0.8 vs. 1.6 events/100 patient-year and 1.4 vs. 1.7 events/ 100 patient-year, respectively). When the benefit of first HHF was incorporated into the Markov model, it was found that adding dapagliflozin treatment incurred a lower first-time treatment cost of HF hospitalization than SoC alone (775 THB/patient vs. $1466 \mathrm{THB} /$ patient or 25.04 USD/patient vs. 47.37 USD/patient). In addition, CKD patients treated with dapagliflozin experienced fewer first HHF compared 
with SoC treatment, with 69 and 130 events per 1000 treated patients over a lifetime horizon, respectively.

Another cost-effectiveness study conducted in the UK using data from the DAPA-CKD clinical trial reported that dapagliflozin in addition to standard therapy was cost-effective treatment for CKD in comparison with standard therapy alone [26]. Our findings were in line with those of the UK study in regard to add-on dapagliflozin being a cost-effective treatment. However, some issues revealed different results. The UK study reported that patients treated with dapagliflozin incurred more total cost over a lifetime horizon, primarily driven by increased drug acquisition costs and increased CKD management costs in earlier CKD stages. This study showed that the total lifetime cost of the dapagliflozin group was lower than that of the SoC group. Delayed CKD progression from dapagliflozin treatment would result in higher numbers of CKD patients in the early CKD stages. The cost of pre-renal replacement therapy is much lower than the cost of dialysis and kidney transplantation in Thailand (Table 2). Therefore, reductions in costs associated with dialysis and kidney transplantation provide important cost-offsets for drug acquisition costs and CKD management costs in earlier CKD stages. Another issue is that this study revealed fewer incremental life-years gained than the UK study ( 0.34 vs. 1.79 years). This might be due to the fact that this study incorporated the ASMR of the Thai population together with the mortality rates from the DAPA-CKD clinical trial. When we removed the ASMR of the Thai population from the analysis, the incremental lifeyears was 1.92 years, which was similar to the findings of the UK study.

The major strength of our study derives from the use of key model parameters such as all costs and some utility data from studies in Thailand. Nonetheless, several limitations are evident. First, the transition probabilities among CKD stages were derived from DAPA-CKD clinical trial data [5], which did not report first HHF in each CKD stage. As a result, we were not able to disaggregate the cost of HF treatment in each CKD stage. Second, we derived the mortality risk of CKD patients based on the median time of 2.4 years from the DAPA-CKD clinical trial [5] and carried forward the constant mortality risk. We addressed this limitation by incorporating the ASMR of the Thai population into the model to reflect the real situation of CKD patients in Thailand. Third, the cohort population in this study had characteristics similar to those in the DAPA-CKD clinical trial, which had baseline eGFR of $25-74 \mathrm{~mL}$ per min per $1.73 \mathrm{~m}^{2}$ of body surface area and a urinary albumin-tocreatinine ratio of $200-5000 \mathrm{mg} / \mathrm{g}$. Therefore, we were unable to confirm the economic benefits in patients with eGFR greater than $74 \mathrm{~mL}$ per min per $1.73 \mathrm{~m}^{2}$ of body surface area or those with urinary albumin-to-creatinine ratio less than $200 \mathrm{mg} / \mathrm{g}$. In addition, the DECLARE study has reported a $47 \%$ reduction in renal specific endpoints $(\geq 40 \%$ decrease in eGFR to $<60 \mathrm{~mL}$ per min per $1.73 \mathrm{~m}^{2}$ of body surface area, new ESRD, or death from renal causes) in type 2 diabetes patients with high cardiovascular risk, and had baseline mean eGFR of $85.2 \mathrm{~mL}$ per min per $1.73 \mathrm{~m}^{2}$ of body surface area $[27,28]$.

\section{CONCLUSIONS}

On the basis of the DAPA-CKD trial, the add-on dapagliflozin results in cost saving compared with SoC alone in Thailand. The benefit of dapagliflozin in delayed CKD progression reduces the requirement for dialysis and KT, which can offset the costs of dapagliflozin and early CKD treatment.

\section{ACKNOWLEDGEMENTS}

Funding. The study was financially supported by AstraZeneca Thailand. The sponsor assisted in literature support and organized the expert meetings. The study sponsor also funded Journal's Rapid Service and Open Access Fees.

Authorship. All named authors meet the International Committee of Medical Journal Editors (ICMJE) criteria for authorship for this article, take responsibility for the integrity of 
the work as a whole, and have given their approval for this version to be published.

Author Contributions. The following describes the contributions each author made to this work: conception and design-Kriengsak Vareesangthip, Chaicharn Deerochanawong, Unchalee Permsuwan; organizing team meeting-Dittaya Thongsuk, Nuch Pojchaijongdee; input data searching-all authors; model and input data verifying-Kriengsak Vareesangthip, Chaicharn Deerochanawong, Unchalee Permsuwan; data analysis-Unchalee Permsuwan; manuscript writing-Kriengsak Vareesangthip, Chaicharn Deerochanawong, Unchalee Permsuwan; approval of final manuscript-all authors.

Disclosures. Chaicharn Deerochanawong, Kriengsak Vareesangthip, Unchalee Permsuwan have nothing to disclose. Dittaya Thongsuk and Nuch Pojchaijongdee are current employees of AstraZeneca Thailand.

Compliance with Ethics Guidelines. This study was based on previously conducted studies and did not include any new studies with human participants or animals performed by any of the authors and was not registered with any clinical trial database.

Data Availability. Data sharing is not applicable as no new datasets were generated or analyzed during this study. No data are deposited in publicly available repositories or published alongside the paper as supplementary material.

Open Access. This article is licensed under a Creative Commons Attribution-NonCommercial 4.0 International License, which permits any non-commercial use, sharing, adaptation, distribution and reproduction in any medium or format, as long as you give appropriate credit to the original author(s) and the source, provide a link to the Creative Commons licence, and indicate if changes were made. The images or other third party material in this article are included in the article's Creative Commons licence, unless indicated otherwise in a credit line to the material. If material is not included in the article's Creative Commons licence and your intended use is not permitted by statutory regulation or exceeds the permitted use, you will need to obtain permission directly from the copyright holder. To view a copy of this licence, visit http://creativecommons.org/licenses/by$\mathrm{nc} / 4.0 /$.

\section{REFERENCES}

1. Lozano R, Naghavi M, Foreman K, Lim S, Shibuya K, Aboyans V, et al. Global and regional mortality from 235 causes of death for 20 age groups in 1990 and 2010: a systematic analysis for the Global Burden of Disease Study 2010. Lancet. 2012;380(9859): 2095-128.

2. Jha V, Garcia-Garcia G, Iseki K, Li Z, Naicker S, Plattner $\mathrm{B}$, et al. Chronic kidney disease: global dimension and perspectives. Lancet. 2013;382(9888):260-72.

3. Liyanage T, Ninomiya T, Jha V, Neal B, Patrice HM, Okpechi I, et al. Worldwide access to treatment for end-stage kidney disease: a systematic review. Lancet. 2015;385(9981):1975-82.

4. Elshahat S, Cockwell P, Maxwell AP, Griffin M, O'Brien T, O'Neill C. The impact of chronic kidney disease on developed countries from a health economics perspective: a systematic scoping review. PLoS ONE. 2020;15(3):e0230512.

5. Heerspink HJL, Stefánsson BV, Correa-Rotter R, Chertow GM, Greene T, Hou FF, et al. Dapagliflozin in patients with chronic kidney disease. N Engl J Med. 2020;383(15):1436-46.

6. Kim KM, Oh HJ, Choi HY, Lee H, Ryu DR. Impact of chronic kidney disease on mortality: a nationwide cohort study. Kidney Res Clin Pract. 2019;38(3): 382-90.

7. Health Statistics, Strategy and Planning Division, Ministry of Public Health. Public Health Statistics A. D.2019. 2021. https://bps.moph.go.th/new_bps/ sites/default/files/statistic62.pdf.

8. Tanvejsilp P, Ngorsuraches S. Defining the scope of health technology assessment and types of health economic evaluation. J Med Assoc Thai. 2014;97(Suppl 5):S10-6.

9. Drug and Medical Supply Information Center. 2021. http://dmsic.moph.go.th. 
10. Krittayaphong R, Permsuwan U. Cost-utility analysis of add-on dapagliflozin treatment in heart failure with reduced ejection fraction. Int J Cardiol. 2021;1(322):183-90.

11. Srinonprasert V, Tantai N, Maneeon S. Cost-effectiveness analysis of SGLT-2 inhibitors for treatment of patients with type 2 diabetes in Thailand. 2020 . http://www.hitap.net/documents/176100.

12. Cha'on U, Wongtrangan $\mathrm{K}$, Thinkhamrop $\mathrm{B}$, Tatiyanupanwong S, Limwattananon C, Pongskul $\mathrm{C}$, et al. CKDNET, a quality improvement project for prevention and reduction of chronic kidney disease in the Northeast Thailand. BMC Public Health. 2020;20(1):1299.

13. Permsuwan U, Dilokthornsakul P, Thavorn K, Saokaew S, Chaiyakunapruk N. Cost-effectiveness of dipeptidyl peptidase-4 inhibitor monotherapy versus sulfonylurea monotherapy for people with type 2 diabetes and chronic kidney disease in Thailand. J Med Econ. 2017;20(2):171-81.

14. The Nephrology Society of Thailand. Thailand renal replacement therapy: year 2016-2019. Bangkok. 2020.

15. Srisubat A, Jiamjariyaporn $\mathrm{T}$, Chanpitakkul $\mathrm{M}$, Leesmidt V, Wisansak W, Promnim S, et al. Costeffectiveness of integrated care in patients with chronic kidney disease stage 3 and 4 compared with standard care in rural communities. J Dept Med Serv. 2017;42(6):54-63.

16. Srisubat A, Sriratanaban J, Ngamkiatphaisan S, Tungsanga K. Cost-effectiveness of annual microalbuminuria screening in Thai diabetics. Asian Biomed. 2014;8(3):371-9.

17. Jesky MD, Dutton M, Dasgupta I, Yadav P, Ng KP, Fenton A, et al. Health-related quality of life impacts mortality but not progression to end-stage renal disease in pre-dialysis chronic kidney disease: a prospective observational study. PLoS ONE. 2016;11(11):e0165675.

18. Li B, Cairns JA, Draper H, Dudley C, Forsythe JL, Johnson RJ, et al. Estimating health-state utility values in kidney transplant recipients and waitinglist patients using the EQ-5D-5L. Value Health. 2017;20(7):976-84.

19. Permsuwan U, Guntawongwan K, Buddhawongsa $P$. Handling time in economic evaluation studies. J Med Assoc Thai. 2014;97(Suppl 5):S50-8.
20. Thavorncharoensap M, Teerawattananon $Y$, Natanant S, Kulpeng W, Yothasamut J, Werayingyong P. Estimating the willingness to pay for a quality-adjusted life year in Thailand: does the context of health gain matter? Clinicoecon Outcomes Res. 2013;5:29-36.

21. Briggs A, Sculpher M. An introduction to Markov modelling for economic evaluation. Pharmacoeconomics. 1998;13(4):397-409.

22. Sugrue DM, Ward T, Rai S, McEwan P, van Haalen HGM. Economic modelling of chronic kidney disease: a systematic literature review to inform conceptual model design. Pharmacoeconomics. 2019;37(12):1451-68.

23. Levin A, Stevens PE, Bilous RW, Coresh J, De Francisco AL, De Jong PE, et al. Kidney Disease: Improving Global Outcomes (KDIGO) CKD Work Group. KDIGO 2012 clinical practice guideline for the evaluation and management of chronic kidney disease. Kidney Int Suppl. 2013;3(1):1-150.

24. Reichel H, Zee J, Tu C, Young E, Pisoni RL, Stengel $\mathrm{B}$, et al. Chronic kidney disease progression and mortality risk profiles in Germany: results from the Chronic Kidney Disease Outcomes and Practice Patterns Study. Nephrol Dial Transplant. 2020;35(5):803-10.

25. McMurray JJV, Wheeler DC, Stefánsson BV, Jongs $\mathrm{N}$, Postmus D, Correa-Rotter R, et al. Effect of dapagliflozin on clinical outcomes in patients with chronic kidney disease, with and without cardiovascular disease. Circulation. 2021;143(5):438-48.

26. McEwan P, Darlington O, Wheeler D, Heerspink H, Bergenheim K, Sanchez JG. POS-335 Cost-effectiveness of dapagliflozin as a treatment for chronic kidney disease: a health-economic analysis of DAPA-CKD. Kidney Int Rep. 2021;6(4):S145-6.

27. Wiviott SD, Raz I, Bonaca MP, Mosenzon O, Kato ET, Cahn A, et al. Dapagliflozin and cardiovascular outcomes in type 2 diabetes. $\mathrm{N}$ Engl J Med. 2019;380(4):347-57.

28. Deerochanawong C, Vareesangthip K, Piyayotai D, Thongsuk D, Pojchaijongdee N, Permsuwan U. Cost-utility analysis of dapagliflozin as an add-on to standard treatment for patients with type 2 diabetes and high risk of cardiovascular disease in Thailand. Diabetes Ther. 2021:1-17. 